\title{
Obstructed labour caused by rare congenital anomaly of foetus: managed by foetal abdominal tapping leading to successful vaginal delivery
}

\author{
Neelam Swaroop, Priya Sharma*, Kalpana Kumari, Shikha Seth
}

\begin{abstract}
Department of Obstetrics \& Gynecology, U.P. Rural Institute Of Medical Sciences \& Research, Saifai, Etawah, Uttar
\end{abstract} Pradesh, India

Received: 21 May 2015

Accepted: 6 June 2015

\author{
*Correspondence: \\ Dr. Priya Sharma, \\ E-mail: dr.sharma.priya@gmail.com
}

Copyright: (c) the author(s), publisher and licensee Medip Academy. This is an open-access article distributed under the terms of the Creative Commons Attribution Non-Commercial License, which permits unrestricted non-commercial use, distribution, and reproduction in any medium, provided the original work is properly cited.

\begin{abstract}
Obstructed labour with high maternal morbidity and mortality is still prevalent in the developing world. The common causes of obstructed labour include contracted pelvis, uterine abnormalities, maternal pelvic tumours, foetal malpresentation, and congenital foetal abnormalities like hydrocephalus, polycystic kidneys/hydronephrosis or locked twins. Here we present a case of rare congenital anomaly of foetus, Prune belly syndrome causing obstructed labour in its course and managed by foetal abdominal tapping leading to successful vaginal delivery. Prune belly syndrome is a rare congenital disease characterized by abdominal wall muscle abnormalities, urinary tract abnormalities and cryptorchidism. It is also associated with cardiovascular, genital, respiratory and other musculoskeletal malformations. Since this patient had no antenatal visit the syndrome was diagnosed after detailed examination of still born foetus.
\end{abstract}

Keywords: Prune belly syndrome, Obstructed labour, Hydronephrosis

\section{INTRODUCTION}

Prune belly syndrome is a rare congenital disease characterized by abdominal wall muscle abnormalities, urinary tract abnormalities and cryptorchidism. It is also associated with cardiovascular, genital, respiratory and other musculoskeletal malformations. ${ }^{1,2}$ The prognosis is usually guarded. Prune belly syndrome can be diagnosed antenatally by ultrasound. But in developing countries, it is not uncommon to have patients presenting to labour room in active labour with no previous antenatal visit and thus such anomalies are detected when they cause obstructed labour. Diagnosis is confirmed postnatally after detailed examination of foetus.

\section{CASE REPORT}

Mrs X, 25 year old, Gravida 2 Para 1, was referred to our labour room, from a primary health centre as a case of obstructed labour with shoulder dystocia. Patient had complain of pain in abdomen following amenorrhea 8 months for which she visited nearby PHC about $8 \mathrm{hrs}$ ago. Patient was in established preterm labour. But the progress of labour was arrested after delivery of baby upto the trunk and thus referred to our tertiary centre. This present pregnancy of the patient was completely unbooked and uninvestigated. There was no significant illness or drug or radiological exposure in present pregnancy.

On examination, patient was in agony and distress. Mild pallor was present and patient was dehydrated and her pulse rate was 94 beats per minute and blood pressure was $110 / 70 \mathrm{mmHg}$. Systemic examination was under normal limit.

The fundal height was 36 weeks palpable, not tender and patient was having good uterine contractions. The foetal heart was not localized with stethoscope. On local 
examination, foetal head and the two upper limbs were lying outside introitus. On vaginal examination, there was slight vulval oedema and the shoulders and chest of the foetus were lying in vagina. There were no masses in the vagina and the cervix was fully dilated through which tense cystic mass was felt. Urgent bedside ultrasound was done, which showed massive distension of foetal abdomen caused by multiseptate cystic masses, probable diagnosis being foetal hydronephrosis and distended urinary bladder. Foetal heart was absent. Informed consent was taken and patient was planned for ultrasound guided trans abdominal drainage after localization of placenta. Taking all aseptic precautions, trans abdominal drainage of cystic masses using wide bore spinal needle and IV. Drip set was done. About 1.5 litres of fluid drained out. After decompression of the foetal abdomen, delivery of the trunk was completed. Congenitally anomalous, still born, male foetus with grossly distended and decompressed abdomen was delivered.

On detailed examination, multiple abnormalities consistent with Prune belly syndrome were noted. The abdomen was distended, of prune-like appearance (wrinkled, loose skin) and anterior abdominal wall musculature was absent, the male genitalia were severely abnormal, small scrotum with cryptorchidism, the chest was significantly small, consistent with lung hypoplasia secondary to anhydramnios, talipes equinovarus was present (Figure 1). Parents refused for the autopsy examination of foetus. Postpartum period of patient was uneventful. Patient was kept on intravenous broad spectrum antibiotics and she was discharged on 5th day post-delivery with proper advice.

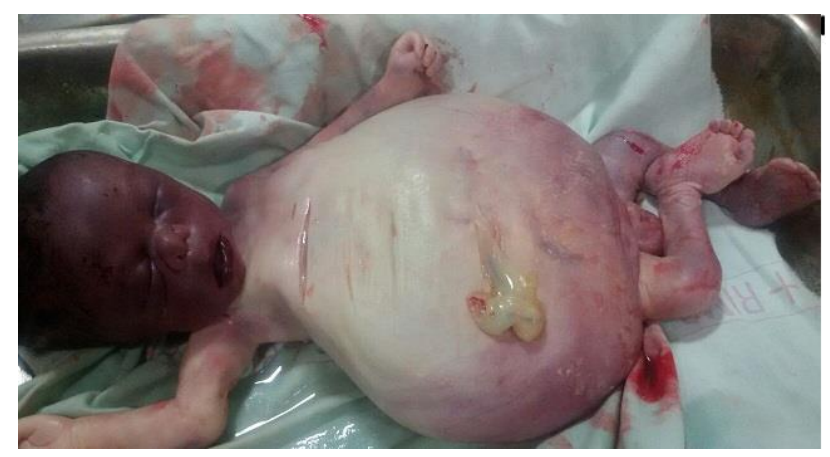

Figure 1: Foetus with prune belly syndrome with enlarged, distended, prune like abdomen, talipes equinovarous, and abnormal external genitilia.

\section{DISCUSSION}

Prune belly syndrome is a rare, genetic birth defect affecting about 1 in 30,000 to 1 in 50,000 births and 3.8 in 100,000 live births. ${ }^{1}$ It is also known as Eagle Barret Syndrome or Obrinsky syndrome or mesenchymal dysplasia or triad syndrome. ${ }^{2}$ About $95 \%$ of those affected are males. ${ }^{3}$
Prune belly syndrome is characterized by a triad of symptoms, characterized by deficient development of abdominal muscles that causes the skin of the abdomen to wrinkle like a prune, bilateral cryptorchidism, abnormalities of the urinary tract such as bilateral gross hydronephrosis, mega ureter and megacystitis. ${ }^{4}$ In addition to classical triad, associated musculoskeletal abnormality, cardiovascular abnormality and genital malformations are noted. ${ }^{1,2}$

Exact etiology is not known. Prune belly syndrome can be diagnosed antenatally by ultrasound. Sonographic findings include oligohydroamnios or anhydramnios, hydroureter, hydronephrosis, a distended urinary bladder and a thin, attenuated abdominal wall. ${ }^{5}$

In new born, the prune like abdomen usually leads to the diagnosis. The prognosis of PBS is usually poor as many infants are either stillborn or die within the first few weeks of life due to pulmonary hypoplasia or renal failure or a combination of congenital anomalies. Chronic failure is the most common complication and found in $25-30 \%$ of cases. ${ }^{4}$ The new-born, who survives, surgery is often required but it will not return the organs to a normal size. Reconstruction surgeries include abdominoplasty, urinary tract reconstruction and orchidopexy. ${ }^{6}$ Diagnosis of prune belly syndrome necessitates thorough orthopedic and cardiological evaluation and treatment because of the high prevalence of associated musculoskeletal and cardiac abnormalities. ${ }^{2,6}$ The routine use of screening for foetal anomalies has resulted in more affected pregnancies being terminated. ${ }^{5}$ If an antenatal diagnosis of urinary obstruction is made, it may be possible to perform vesicoamniotic shunting and intrauterine surgery to prevent the development of prune belly syndrome. The results seem promising. 6,7

But in developing countries the scenario is different. A substantial percentage of antenatal women especially from rural areas do not get antenatal check-ups and ultrasonography done and the anomaly in foetus goes undetected, as happened in our case. Such antenatal women visit the health centre only when the labour sets in and labour course is obstructed and nothing can be done to prevent the perinatal mortality.

Similar case was reported by Cardoso et al which was also managed by foetal abdominal tapping. ${ }^{8}$

Funding: No funding sources

Conflict of interest: None declared

Ethical approval: Not required

\section{REFERENCES}

1. Routh JC, Haung L, Retik AB, Nelson CP. Contemporary epidemiology of newborn males with prune belly syndrome. Urology. 2010;76:44. 
2. Nunn IN, Stephens FD. The triad syndrome: a composite anomaly of the abdominal wall, urinary system and testes. J Urol.1961;86:782.

3. Druschel CM. A descriptive study of prune belly in New York State 1983 to 1989. Arch Pediatr Adolesc Med.1995;149:70.

4. Tagore KR, Ramineni AK, Vijaya Lakshmi AR, Bhavani N. Prune Belly syndrome. Case Rep Pediatr. 2011:1-3.

5. Ellison L, Cendron M, Ornvold K, et al. Early diagnosis of fetal bladder outlet obstruction. J Pediatr Surg. 2000;35:513.

6. Denes FT, Arap MA, Giron AM, et al. Comprehensive surgical treatment of prune belly syndrome: 17 years' experience with 32 patients. Urology. 2004;64:789.
7. Biard JM, Johnson MP, Carr MC, et al; Long-term outcomes in children treated by prenatal vesicoamniotic shunting for lower urinary tract obstruction. Obstet Gynecol. 2005;106(3):503-8.

8. Cardoso PM, Surve S, Pednecar G, Mello AD. A Rare Fetal Anomaly Causing Obstructed Labour. Journal of Evolution of Medical and Dental Sciences. 2014;3(56):12829-33.

Cite this article as: Swaroop N, Sharma P, Kumari $\mathrm{K}$, Seth S. Obstructed labour caused by rare congenital anomaly of foetus: managed by foetal abdominal tapping leading to successful vaginal delivery. Int J Reprod Contracept Obstet Gynecol 2015;4:1220-2. 